\title{
Ironless transducer for measuring the mechanical properties of porous materials
}

\author{
Olivier Doutres, Nicolas Dauchez, ${ }^{\text {a) }}$ Jean-Michel Genevaux, \\ Guy Lemarquand, and Sylvain Mezil \\ Laboratoire d'Acoustique de l'Université du Maine, UMR CNRS 6613, Avenue Olivier Messiaen, \\ 72085 Le Mans Cedex 9, France
}

(Received 18 January 2010; accepted 28 March 2010; published online 3 May 2010)

\begin{abstract}
This paper presents a measurement setup for determining the mechanical properties of porous materials at low and medium frequencies by extending toward higher frequencies the quasistatic method based on a compression test. Indeed, classical quasistatic methods generally neglect the inertia effect of the porous sample and the coupling between the surrounding fluid and the frame; they are restricted to low frequency range $(<100 \mathrm{~Hz})$ or specific sample shape. In the present method, the porous sample is placed in a cavity to avoid a lateral airflow. Then a specific electrodynamic ironless transducer is used to compress the sample. This highly linear transducer is used as actuator and sensor; the mechanical impedance of the porous sample is deduced from the measurement of the electrical impedance of the transducer. The loss factor and the Young's modulus of the porous material are estimated by inverse method based on the Biot's model. Experimental results obtained with a polymer foam show the validity of the method in comparison with quasistatic method. The frequency limit has been extended from $100 \mathrm{~Hz}$ to $500 \mathrm{~Hz}$. The sensitivity of each input parameter is estimated in order to point out the limitations of the method. (c) 2010 American Institute of Physics. [doi:10.1063/1.3398419]
\end{abstract}

\section{INTRODUCTION}

Porous materials, such as open cell polymer foams or mineral wools, are widely used for acoustical comfort design in building or transport applications. Due to their high porosity (usually $>90 \%$ ) and an adequate pore size (around 10 to $100 \mu \mathrm{m}$ ), they lead to sound absorption by viscothermal dissipation. ${ }^{1}$ When attached to a vibrating structure, they also bring (i) structural damping due to mechanical coupling between the vibrating structure and the frame ${ }^{2}$ and (ii) sound transmission reduction or amplification of the structure due to interaction between the frame motion and the saturating fluid. ${ }^{3}$ Their efficiency can thus be greatly influenced by the viscoelastic properties of the frame $e^{4,5}$ that is accounted for in the Biot-Allard theory. ${ }^{1,6}$ In order to achieve a proper design of acoustical treatment, the viscoelastic properties of porous materials have to be known.

Classical methods to measure the viscoelastic properties of the frame can be sorted in two groups: ${ }^{7}$ the quasistatic methods neglect the inertia effects and give relevant information in the low frequency range before the first resonance of the system ${ }^{8-11}$ (usually for $f<100 \mathrm{~Hz}$ ) and the dynamic methods are based on the vibration study of a porous layer, ${ }^{12-14}$ or of a structure that includes a porous layer, ${ }^{15,16}$ and give information at the resonance frequencies of the structure. Most of the existing methods are carried out in ambient conditions because "in vacuum" conditions lead to some experimental issues: the experimental setup is heavier,

\footnotetext{
${ }^{a)}$ Institut Supérieur de Mécanique de Paris-SUPMECA Paris, LISMMA, 3
} rue Fernand Hainault, 93407 Saint Ouen Cedex, France. the frame of some types of acoustical material can be altered, and the temperature has to be slightly controlled. However, the methods carried out in ambient conditions neglect the effects of the coupling between the frame and the saturating fluid, and the material is considered as in vacuum. Pritz, ${ }^{15,16}$ Ingard ${ }^{17}$ and Rice and Göransson ${ }^{18}$ carried out frame compressibility measurements in air and in vacuum using the resonant method of Fig. 1(a). In this configuration, the porous sample is placed between a vibrating base and an additional mass; the structure is supposed to behave as a springmass system. It is shown that the frequency response of the mass with respect to the shaker table motion is considerably damped when air is present. This difference is due to the presence of a lateral airflow (perpendicular to the imposed displacement) pumped in and out of the material during sinusoidal compression. The evaluation of the Young's modulus is hardly affected by the presence of the air, but the apparent loss factor of the material is greatly overestimated.

The effect of air on a quasistatic measurement, such as proposed by Mariez ${ }^{8}$ [Fig. 1(b)], has also been studied numerically by Etchessahar ${ }^{14}$ and Danilov et al. ${ }^{19}$ In this configuration, the porous sample is placed between a vibrating base and a impervious rigid wall, and the mechanical impedance is measured. It is shown that both real and imaginary parts of this impedance can be greatly influenced by the presence of air for thin samples or materials having a large airflow resistivity. Tarnow ${ }^{10}$ proposed an analytical correction that accounts for this influence on the measurement of the force transmitted by the porous sample to the rigid wall for cylindrical samples. In a previous paper, ${ }^{20}$ the authors 


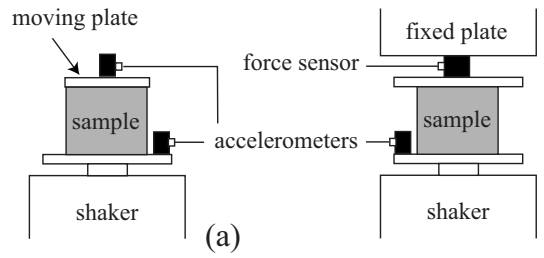

(b)

FIG. 1. Classical measurement setup: (a) seismic test, measurement of the ratio of the velocities between the shaker table and the top mass, and (b) compression test, measurement of the ratio of the force transmitted by the porous sample to the fixed plate $F$ on the velocity of the shaker table $v$.

investigated the feasibility to extend the quasistatic compression method toward higher frequencies by mean of

- a cavity where the sample is set up in order to reduce the air-pumping effects and

- an electrodynamic loudspeaker as actuator and sensor to simplify the measurement setup.

This method is in good agreement with the quasistatic one, but the frequency range was limited below the first frequency resonance of the loudspeaker by non linearities of the transducer.

In the present paper, the method is extended toward medium frequencies by using a specific electrodynamic transducer devoid of major nonlinearities. In a first part, the transducer design is described and the measurement procedure is detailed. Then results are given for one polymer foam. The sensitivity of each input parameter is estimated in order to point out the limitations of the method.

\section{MEASUREMENT SETUP}

The electromechanical setup to measure the porous mechanical properties is shown in Fig. 2. The electrodynamic transducer used to compress the sample is mounted between two cavities. It is made of a voice-coil motor such as those used in traditional electrodynamic loudspeakers. However, this motor is ironless, ${ }^{21,22}$ and the viscoelastic suspension is replaced by ferrofluid seals ${ }^{23}$ in order to vanish the major electrical and mechanical nonlinearities, ${ }^{24,25}$ respectively. Furthermore, the motor is constituted by a stack of three permanent magnet rings $20 \mathrm{~mm}$ high with an inner diameter

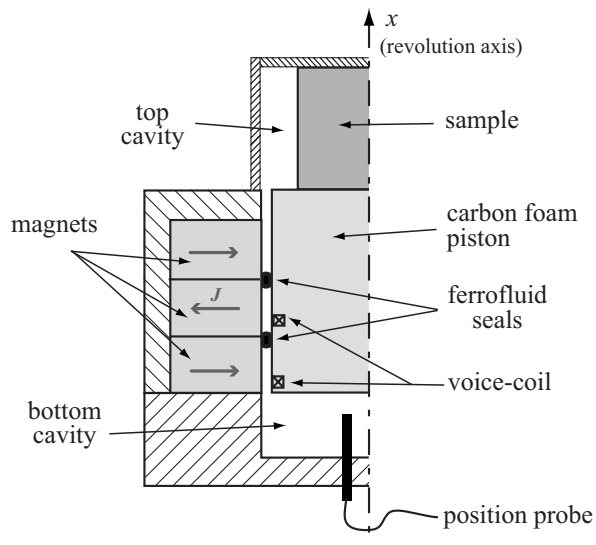

FIG. 2. Measurement setup for the electrodynamic method. $x$ axis is oriented in the direction opposite to the gravity field.

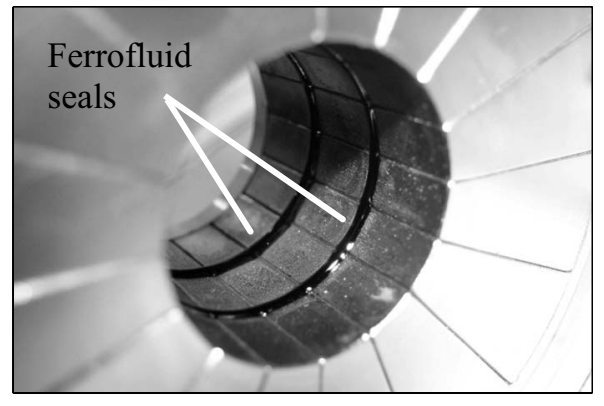

FIG. 3. Magnet assembly with two ferrofluid seals without moving piston.

of $49.7 \mathrm{~mm}$. Each ring is an assembly of 16 single tiles (Fig. 3) of $\mathrm{Nd}_{2} \mathrm{Fe}_{14} \mathrm{~B}$ with a magnetization of $J=1.4 \mathrm{~T}$. The rings are radially magnetized with successive opposite directions (Fig. 2) to avoid a magnetic field leakage. ${ }^{26}$ Figure 4 shows an analytical simulation ${ }^{27}$ of the radial magnetic field seen by the coils, i.e., at a distance of $0.3 \mathrm{~mm}$ of the magnets. It is shown that the magnetic structure allows a high magnetic field (around $0.7 \mathrm{~T}$ ), which is constant over $10 \mathrm{~mm}$ on the height of each ring. Thus, the electrodynamic motor can be used to apply a static compression of $10 \mathrm{~mm}$ and preserve the same properties. The inner face of the motor presents also a strong gradient of the magnetic field where the magnetic field reverses, i.e., at the interface between two rings. These regions, where the magnetic pressure is the most important, $^{23}$ define the position of ferrofluid seals, which guide the piston along the " $x$ " direction ${ }^{28,29}$ and ensure the airtightness between the two cavities (Fig. 3).

The moving piston is a cylindrical monobloc structure made out of carbon foam, coated with a varnish to avoid the penetration of ferrofluid into the carbon foam. It is $70 \mathrm{~mm}$ high with a diameter of $49.1 \mathrm{~mm}$. Because of its particular mechanical properties, this material allows a very stiff and light piston (Young's Modulus: $70 \mathrm{MPa}$; Bulk density: $30 \mathrm{~kg} \mathrm{~m}^{-3}$; and Poisson coefficient: 0.3 ). The first natural frequency of the monobloc piston in free conditions is situated over $7 \mathrm{kHz}$. This is far from the frequency range of measurement $(100 \mathrm{~Hz}-1 \mathrm{kHz})$. The piston is thus considered as rigid. Note that the piston material is an electrical isolator and is transparent to the magnetic field.

Two ridges are present on the outer surface of the piston to receive the coil windings. The upper voice-coil is designed to apply a small amplitude dynamic stress to the porous

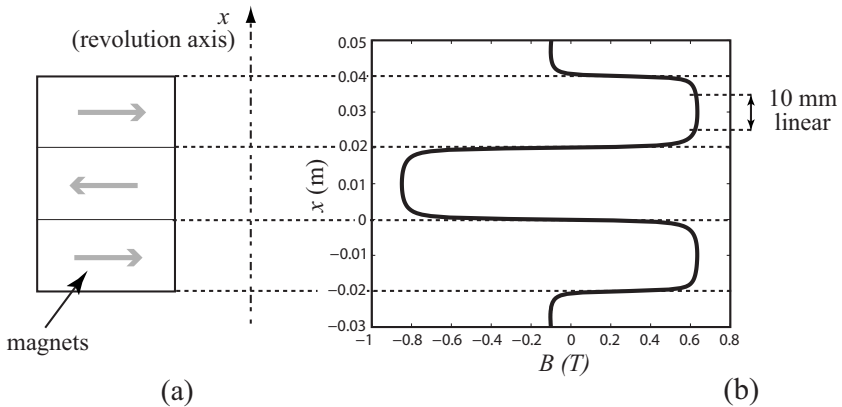

FIG. 4. (a) 2D axisymmetric representation of the magnetic structure; (b) Radial magnetic field seen by the coil (at a distance of $0.3 \mathrm{~mm}$ of the magnets). 


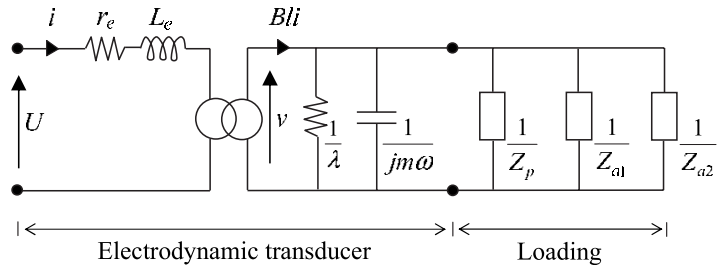

FIG. 5. Electrical equivalent model of the measurement setup.

sample. Its electrical impedance is used to deduce the mechanical impedance of the sample. The lower voice-coil supports a direct current to apply a static strain to the sample. This strain is monitored by a position probe. The diameter and the length of the coil have been determined so that a porous material with Young's modulus of $400 \mathrm{kPa}$ can be compressed with a static strain of $2 \%$. For softer materials such as glass wool, the dimensions of the voice-coil motor allow a maximum static compression of $10 \mathrm{~mm}$.

Note that since the ferrofluid seals have no stiffness in the axial direction, unlike rubber suspension and spider in traditional loudspeakers, the axial stiffness is only due to the sample and to the air cavities loading the piston. Finally, the diameter of the sample has to be smaller than the diameter of the top cavity to avoid lateral friction.

\section{PRINCIPLE OF MEASUREMENT}

The electrodynamic transducer is used to apply static and dynamic stresses to the porous sample. From the measurement of the electrical impedance $Z_{v c}$ of the upper voicecoil and a linear model of the transducer behavior, the mechanical impedance $Z_{p}=F / v$ at the porous/piston interface is determined. $F$ is the force applied to the porous sample, and $v$ is the velocity of the piston. The mechanical properties of the porous material, Young's modulus $E$ and loss factor $\eta$, are then determined from the mechanical impedance of the sample by reversing a poroelastic model based on the BiotAllard theory. ${ }^{1}$

\section{A. Transducer modeling}

The transducer modeling is identical to the one used in a previous paper. ${ }^{20}$ However, since the motor is ironless and the viscoelastic suspension is replaced by ferrofluid seals, both the compliance of the loudspeaker suspensions $C_{m s}$ and the shunting parallel resistance $r_{f}$ are considered equal to zero. This model is now briefly presented.

The electrodynamic transducer is described by an equivalent electrical circuit. The development of this model is described in detail by Thiele and Small. ${ }^{30-32}$ This low frequency model is valid under the first resonance frequency of the piston (here $7 \mathrm{kHz}$ in free conditions). The moving piston is thus considered as rigid and having only one degree of freedom in the axial direction.

Figure 5 is the analogous circuit for the setup of Fig. 2, with

- $U$, input voltage of the dynamic voice-coil,

- $i$, current passing through the dynamic voice-coil,

- $r_{e}$, dc resistance of the dynamic voice-coil,
- $L_{e}$, inductance of the dynamic voice-coil,

- $B$, magnetic flux density in air gap,

- $l$, length of voice-coil in magnetic field,

- $\lambda$, viscous damping coefficient of the moving piston,

- $m$, mass of the moving piston including voice-coils,

- $Z_{a 1}$, mechanic impedance of top air cavity surrounding the porous sample,

- $Z_{a 2}$, mechanic impedance of bottom air cavity, and

- $Z_{p}$, mechanic impedance of porous sample.

The mechanical impedances of air cavities are obtained by considering the sum of both forth and back propagating acoustical waves in the cavity, giving ${ }^{1}$

$$
\begin{aligned}
& Z_{a 1}=-j Z_{c} S^{\prime} \operatorname{cotg} \delta_{0} d_{1}, \\
& Z_{a 2}=-j Z_{c} S \operatorname{cotg} \delta_{0} d_{2},
\end{aligned}
$$

where $j$ is the square root of $-1, \omega$ is the circular frequency of the excitation, $\rho_{0}$ is the air density, $c$ is the velocity of sound in air, $\delta_{0}=\omega / c$ is the wavenumber of sound in air, $Z_{c}=\rho_{0} c$ is the characteristic impedance of air, $d_{1}$ and $d_{2}$ are the length of the top and bottom cavities, respectively, $S^{\prime}$ is the surface area of the piston in contact with air in the top cavity, and $S$ the surface area of the piston in contact with the bottom cavity. Note that in the low frequency range, the two air cavities can be considered as simple compliances, and Eqs. (1) and (2) may be simplified as

$$
\begin{aligned}
& Z_{a 1}^{l f}=\frac{\gamma P_{0} S^{\prime}}{j \omega d_{1}}, \\
& Z_{a 2}^{l f}=\frac{\gamma P_{0} S}{j \omega d_{2}},
\end{aligned}
$$

where $\gamma P_{0}$ is the adiabatic bulk modulus of the fluid with $\gamma$ as the ratio specific heats and $P_{0}$ as the atmospheric pressure.

From circuit analysis in Fig. 5, the electrical impedance is given by

$$
Z_{v c}=\frac{U}{i}=Z_{e}+\frac{(B l)^{2}}{Z_{m}+\left[Z_{p}+Z_{a 1}+Z_{a 2}\right]},
$$

with

$$
\begin{aligned}
& Z_{e}=r_{e}+j \omega L_{e}, \\
& Z_{m}=\lambda+j \omega m .
\end{aligned}
$$

Hence, the mechanical impedance of the sample can be derived from the measurement of $Z_{v c}$, the properties of the transducer, and of the two air cavities as

$$
Z_{p}=\frac{(B l)^{2}}{Z_{v c}-Z_{e}}-\left(Z_{m}+Z_{a 1}+Z_{a 2}\right) \text {. }
$$

\section{B. Determination of the transducer properties}

The properties of the transducer are determined from the measurement of the electrical impedance $Z_{v c 0}$ without porous sample in the top cavity. In that case, the equivalent electrical circuit model gives 


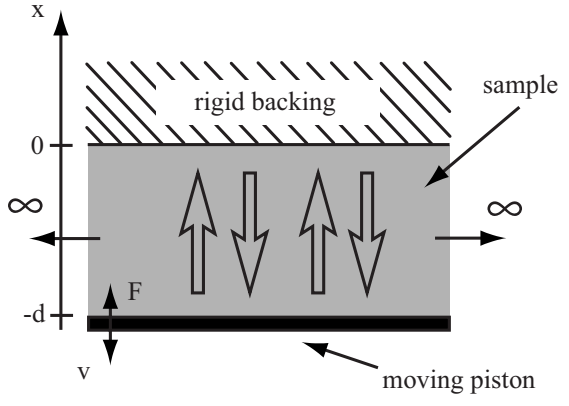

FIG. 6. 1D model of a porous layer compressed on a rigid backing.

$$
Z_{v c 0}=Z_{e}+\frac{(B l)^{2}}{Z_{m}+\left[Z_{a 10}+Z_{a 2}\right]},
$$

with $Z_{a 10}=-j Z_{c} S \operatorname{cotg} \delta_{0} d_{1}$ [Eq. (1) with $S^{\prime}=S$ ]. The model is fitted on the measurement to get $r_{e}, L_{e}, \lambda, m$, and $B l$.

\section{Calculation of the material mechanical properties}

The mechanical properties of the porous sample are determined by fitting a poroelastic model based on the BiotAllard theory on the impedance $Z_{p}$, determined from the measurement of $Z_{v c}$ [Eq. (5)]. In the considered model, the material is isotropic, and the displacements of the frame and air are one-dimensional (1D) (Fig. 6); the porous material is considered as infinite in the lateral directions, and the effects of the boundary conditions are neglected. This assumption is discussed in the next section.

The mechanical impedance can be derived analytically from the calculation of the total stress $\sigma_{x x}^{t}$ applied by the porous sample to the vibrating piston (Fig. 6),

$$
Z_{p}^{t h}(\omega)=\frac{F}{v}=\frac{S_{p} \sigma_{x x}^{t}(-d)}{J \omega u_{w}},
$$

with $S_{p}$ as the surface area of the porous sample in contact with the vibrating piston $\left(S_{p}=S-S^{\prime}\right), u_{w}$ as the amplitude of the displacement imposed by the piston, and $d$ as the sample thickness. Note that the porous material is considered as infinite in the lateral directions in the poroelastic model, but the lateral dimensions are taken into account through the area of the sample. According to the Biot-Allard theory, ${ }^{1,6}$ three waves may propagate in a porous media: two compressional waves and a shear wave. In this work, the shear wave is not excited, and only the two compressional waves are considered. These waves are characterized by a complex wavenumber $\delta_{i}(i=1,2)$ and a displacement ratio $\mu_{i}$. This ratio indicates in which medium the waves mainly propagate. The total stress is thus the sum of the stress exerted by the fluid and solid phases characterized by these two waves as

$$
\begin{aligned}
\sigma_{x x}^{t}(-d)= & \sigma_{x x}^{s}(-d)+\sigma_{x x}^{f}(-d) \\
= & {\left[(\widetilde{P}+\widetilde{Q})+\mu_{1}(\widetilde{R}+\widetilde{Q})\right] \delta_{1} \cos \left(\delta_{1} d\right) D_{1} } \\
& +\left[(\widetilde{P}+\widetilde{Q})+\mu_{2}(\widetilde{R}+\widetilde{Q})\right] \delta_{2} \cos \left(\delta_{2} d\right) D_{2} .
\end{aligned}
$$

In these equations, $\widetilde{R}$ is the bulk modulus of the fluid phase and $\widetilde{Q}$ quantifies the potential coupling between the solid and fluid phases. The expression of these two last coefficients can
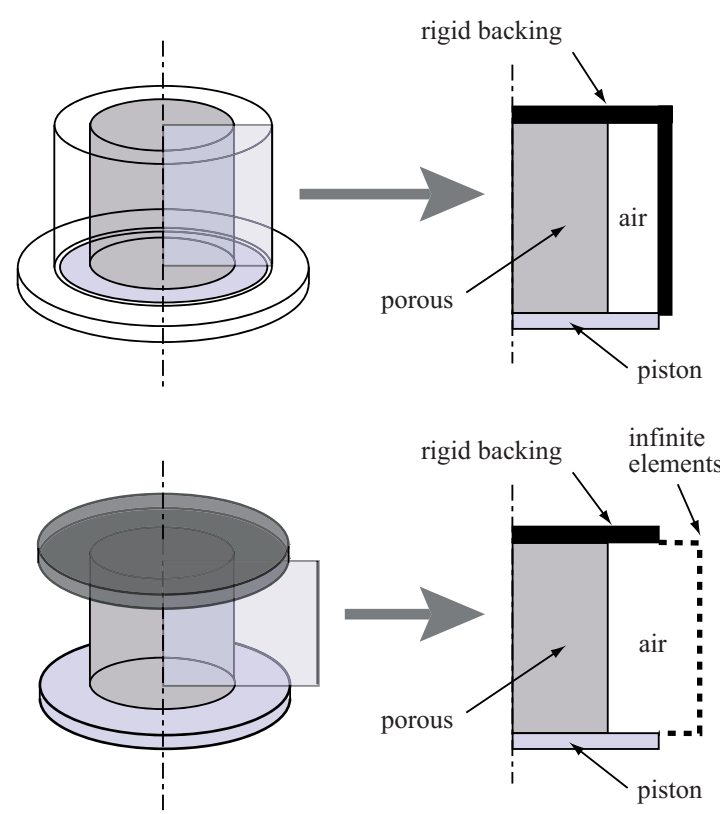

FIG. 7. (Color online) 2D axisymmetric representation of a porous sample compressed between two rigid plates: (a) sample in a cavity and (b) sample in free space.

be found in Ref. 1. $D_{1}$ and $D_{2}$ are the amplitude coefficients of the two compressional waves and can be determined from the boundary conditions applied to the sample. Here, the displacement is null at $x=0$ and is equal to the one of the piston at $x=-d$, which gives

$$
\begin{aligned}
& D_{1}=\frac{u_{w}\left(\mu_{2}-1\right)}{\sin \left(\delta_{1} d\right)\left(\mu_{1}-\mu_{2}\right)}, \\
& D_{2}=\frac{u_{w}\left(1-\mu_{1}\right)}{\sin \left(\delta_{2} d\right)\left(\mu_{1}-\mu_{2}\right)} .
\end{aligned}
$$

\section{Influence of the presence of the top cavity}

As mentioned previously, the top cavity is used to avoid the air-pumping effect. This effect has been investigated in the configuration of Fig. 1(b), and it has been shown that this effect yields to an overestimation of the loss factor of the porous material. ${ }^{7,9,10,19}$ Note that the setup in Fig. 1(b), called quasistatic, is generally used in the low frequency range $(<100 \mathrm{~Hz})$. The coupling between the frame and the air is then neglected, and the porous material is modeled as a monophasic solid, which has the stiffness and structural dissipation of the frame and the density of the porous material.

The aim of this section is to show the efficiency of the top cavity, allowing the use of a 1D analytical modeling for inverse method instead of finite element method (FEM) method that leads to heavier computation. The mechanical impedances given by the two configurations presented in Figs. 1(b) and 2 have been simulated by FEM using the code MSC ACTRAN. The two considered configurations, with and without cavity, are depicted in Fig. 7. The porous material is a polymer foam of thickness $20 \mathrm{~mm}$. Its characteristics are given in Table II. The Poisson coefficient $\nu$ is here set equal to 0 . The diameter of the sample is $44 \mathrm{~mm}$, and in the case of the configuration in Fig. 7(a), the inner diameter of the cavity 

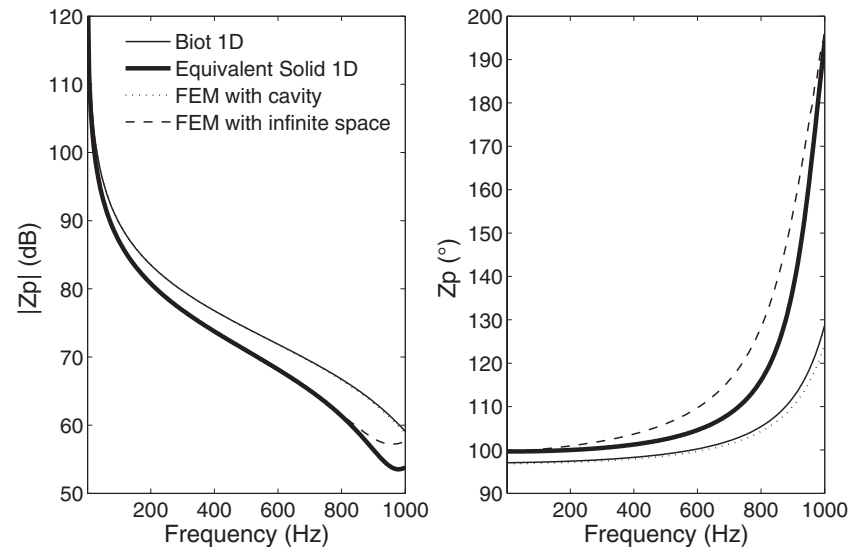

FIG. 8. Simulation of the mechanical impedance of foam A: $L=20 \mathrm{~mm}$, $E=280 \mathrm{kPa}, \eta=0.17$, and $\nu=0$.

is $50 \mathrm{~mm}$; the air layer surrounding the porous sample is $3 \mathrm{~mm}$ thick.

FEM and 1D simulations of the two mechanical impedances are presented in Fig. 8. The hypotheses for the 1D simulations are that in the case of the porous placed in the cavity, the 1D Biot model is used and when the porous is placed in free space, the porous material is considered as a simple monophasic elastic solid. Figure 8 shows that the 1D Biot simulation (thin continuous line) is in good agreement with the numerical one (dotted line); the $3 \mathrm{~mm}$ air gap around the sample has relatively insignificant effect. In the case without cavity, the simple 1D solid model (thick continuous line) allows to describe the module of the impedance under the first resonance frequency (around $900 \mathrm{~Hz}$ ) in comparison with FEM model (dashed line), but a significant discrepancy can be observed in the estimation of the impedance phase.

In order to show the influence of these discrepancies on the result of the inverse method, FEM simulations are now considered as input, and the analytical models are fitted to determine back the viscoelastic properties of the material. The initial viscoelastic properties used in the numerical modeling were considered frequency independent: $E=280 \mathrm{kPa}$ and $\eta=0.17$. Results of the fitted viscoelastic properties are
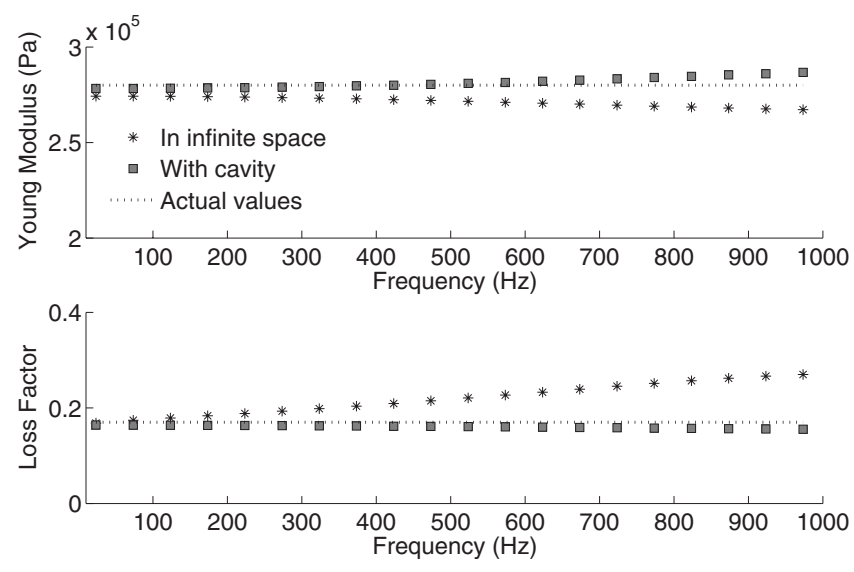

FIG. 9. Viscoelastic properties of foam A determined by the quasistatic method and the electroacoustic method (real values: $E=280 \mathrm{kPa}, \eta=0.17$, and $\nu=0)$.
TABLE I. Properties of the transducer and their standard uncertainties.

\begin{tabular}{lcccc}
\hline \hline $\begin{array}{l}r_{e} \\
(\Omega)\end{array}$ & $\begin{array}{c}L_{e} \\
(\mu \mathrm{H})\end{array}$ & $\begin{array}{c}B l \\
\left(\mathrm{~N} \mathrm{~A}^{-1}\right)\end{array}$ & $\begin{array}{c}m \\
(\mathrm{~g})\end{array}$ & $\begin{array}{c}\lambda \\
\left(\mathrm{N} \mathrm{s} \mathrm{m}^{-1}\right)\end{array}$ \\
\hline 53.66 & 862.9 & 8.56 & 9.832 & 13.85 \\
0.06 & 20 & 0.05 & 0.05 & 0.5 \\
\hline
\end{tabular}

given in Fig. 9. The viscoelastic properties determined in the configuration with the top cavity are in good agreement with the actual values. In the configuration without cavity, we verify here that the loss factor of the frame can be greatly overestimated. Indeed, the coupling between the surrounding fluid and the frame is not taken into account in the 1D model.

It has been verified that (i) when a cavity is present, the simple 1D Biot model can be used to determine the viscoelastic properties of the foam from the mechanical impedance because the air-pumping effects are not significant and (ii) when a cavity is not present, the use of a 1D solid model leads to an overestimation of the porous material loss factor increasing with frequency because the air-pumping effects are not taken into account in the model. This validates the benefit of using a cavity to extend the frequency range toward higher frequencies without requiring the use of FEM modeling.

\section{E. Uncertainties}

The combined uncertainty due to variables entering in the determination of the viscoelastic properties has been evaluated using the classical method of the propagation of errors formulas, ${ }^{33}$ neglecting covariance of the parameters,

$$
u_{c}^{2}=\sum_{i=1}^{N} C_{i},
$$

with $C_{i}$ as the contribution of each parameter to the combined uncertainty defined by

$$
C_{i}=\left(\frac{\partial f}{\partial x_{i}}\right)^{2} u^{2}\left(x_{i}\right),
$$

where $u\left(x_{i}\right)$ is the standard uncertainty related to the variable $x_{i}$ and $f$ is the function relying $x_{i}$ to the parameter either $E$ or $\eta$. The $N$ variables considered are the electrical impedance $Z_{v c}$ and $Z_{v c 0}$ with and without sample, respectively, those of the transducer $\left(r_{e}, L_{e}, m, \lambda, B l\right)$, and those of the porous material, which are used for the inverse identification $\left(\sigma, \phi, \alpha_{\infty}, \Lambda, \Lambda^{\prime}, \rho_{1}\right)$. The partial derivatives are estimated numerically. Uncertainties are defined by the variance $s_{i}$

TABLE II. Properties of the polymer foam with uncertainties.

\begin{tabular}{lccc}
\hline \hline & & Value & $\begin{array}{c}\text { Uncertainty } \\
(\%)\end{array}$ \\
\hline Airflow resistivity & $\sigma$ & $57 \mathrm{kN} \mathrm{s} / \mathrm{m}^{4}$ & 2 \\
Porosity & $\phi$ & 0.97 & 1 \\
Tortuosity & $\alpha_{\infty}$ & 1.54 & 10 \\
Viscous length & $\Lambda$ & $11 \mu \mathrm{m}$ & 20 \\
Thermal length & $\Lambda^{\prime}$ & $30 \mu \mathrm{m}$ & 20 \\
Frame density & $\rho_{1}$ & $46 \mathrm{~kg} / \mathrm{m}^{3}$ & 1 \\
\hline \hline
\end{tabular}



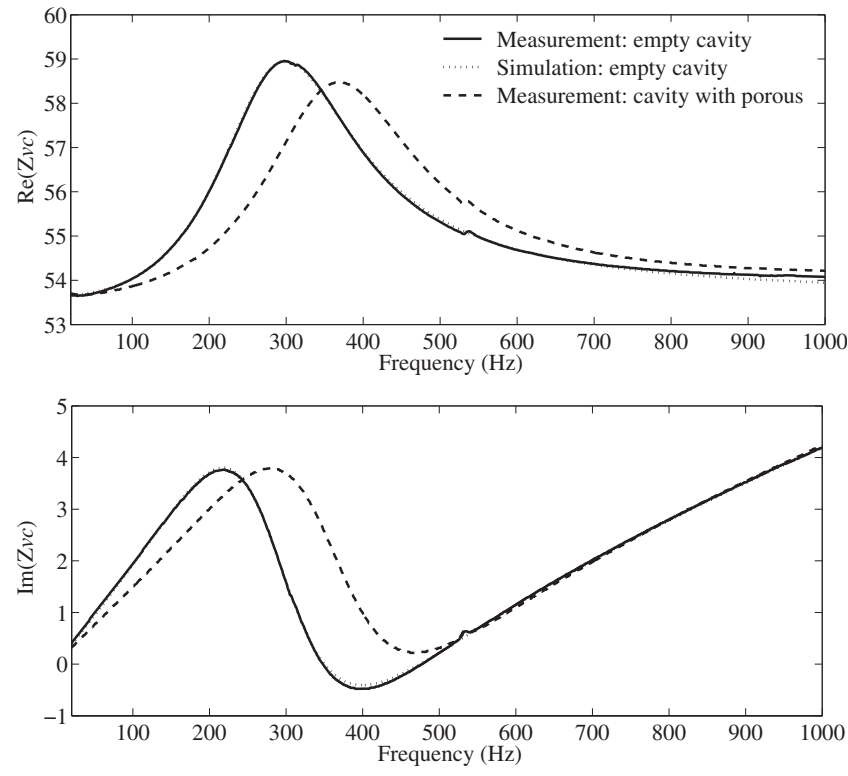

FIG. 10. Electrical impedance $Z_{v c}$ of the transducer.

determined from repeatability tests $q_{i}$ for a type A of $n$ evaluations,

$$
s_{i}^{2}=\frac{1}{n-1} \sum_{j=1}^{n}\left(q_{j}-\bar{q}\right)^{2},
$$

where $\bar{q}$ is the average value of the $q_{i}$. The uncertainty on $Z_{v c}$ is $10^{-3} \Omega$. The uncertainty of the others variables are given in Tables I and II. The expanded uncertainties $U=k u_{c}$ is obtained considering the coverage factor $k=2$.

\section{RESULTS}

\section{A. Properties of the transducer}

The properties of the transducer determined from the measurement of the electrical impedance when the top cavity is empty are given in Table I. Figure 10 shows the measured curve and the simulated curve obtained with the fitted parameters. It can be noticed that the major discrepancy appears on the real part of $Z_{v c}$ above $800 \mathrm{~Hz}$.

\section{B. Properties of the porous sample}

Measurements are carried out with a sample of polymer foam whose diameter is $44.5 \mathrm{~mm}$ and thickness is $19.0 \mathrm{~mm}$ at rest. The characteristics of the foam presented in Table II have been measured in our laboratory ${ }^{34}$ with dedicated benches. Their typical standard uncertainties are also given. ${ }^{35}$ The electrical impedance measured for a compression rate of $1 \%$ is presented in Fig. 10. Compared to the empty cavity measurement, the resonance pick of the moving rigid piston is higher in frequency and lower in amplitude.

The mechanical impedance determined from Eq. (8) and the simulation derived from Eq. (10) are shown in Fig. 11. The continuous line is the theoretical impedance of the empty cavity $Z_{a 10}^{\text {th }}$ considered as simple constant compliance [Eq. (3) with $\left.S^{\prime}=S\right]$. The theoretical impedance of the sample $Z_{p}^{\text {th }}$ (dotted line) shows the same trends since the complex Young's modulus is also considered constant [value
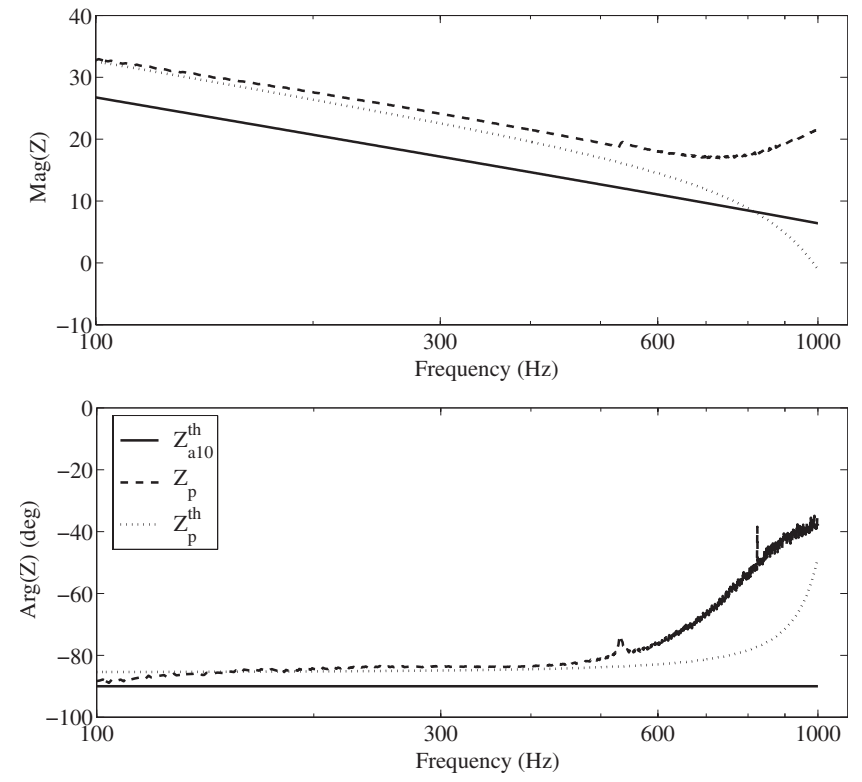

FIG. 11. Magnitude and phase of the mechanical impedance: $Z_{a 10}^{\text {th }}$ simulation of the empty top cavity [Eq. (3)], $Z_{p}^{\text {th }}$ simulation of the sample, and $Z_{p}$ measurement of the sample.

estimated at $5 \mathrm{~Hz}$ using the compression quasistatic method ${ }^{11}$ (Fig. 12)]. Below $600 \mathrm{~Hz}$, the dynamic behavior of the sample can be observed. The measured impedance of the sample (dashed line) differs slightly from theoretical plot since viscoelastic properties of the foam are frequency dependant. Above $600 \mathrm{~Hz}$, the discrepancy is more pronounced: measurements of the mechanical impedance $Z_{p}$ becomes erroneous because the model of the transducer is no more valid.

Viscoelastic properties according to frequency are finally determined by fitting the poroelastic model on the measurement at each frequency. Figure 12 gives the Young's modulus and the loss factor evaluated with the classical compres-
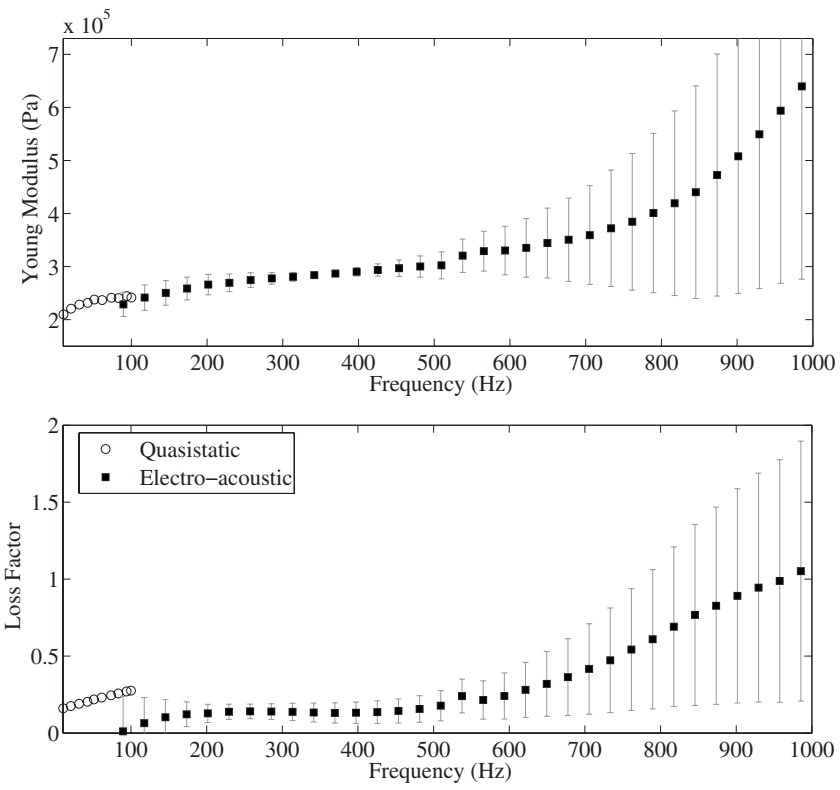

FIG. 12. Measured Young's modulus (top) and loss factor (bottom) of the polymer foam. 
sion quasistatic method in Fig. 1 and the proposed electrodynamic method for the polymer foam. The vertical lines indicate the two-sided confidence interval at $95 \%$ on the

values of $E$ and $\eta$, estimated from the standard uncertainties.

As shown in Fig. 12, good agreements are found in the evaluation of the Young's modulus: it is around $220 \mathrm{kPa}$ at $100 \mathrm{~Hz}$ and increases with frequency. The loss factor is found around 0.3 by the quasistatic method at $100 \mathrm{~Hz}$ and around 0.1 by the electrodynamic method between 200 and $500 \mathrm{~Hz}$. This difference can be explained by the very high airflow resistivity of the sample, and then the important airpumping effect when the sample is placed in free space. This demonstrates experimentally the efficiency of the top cavity for this kind of material.

\section{Discussion}

The main issue of the proposed method comes from the fact that the transducer is used as a sensor with a need of an exact modeling of its behavior [Eq. (8)]. It has the advantage to simplify the experimental setup compared to the classical compression method, but on the other hand, it requires (i) a transducer as linear as possible ${ }^{20}$ and (ii) an electrical impedance measurement devoid of any exterior disturbances (electrical contacts, effect of the temperature, etc.).

Indeed, the quick increase in the viscoelastic parameters above $600 \mathrm{~Hz}$ (Fig. 12) is mainly due to the fact that the simple Thiele and Small model (Sec. III A) does not allow to perfectly describe the behavior of the transducer in this higher frequency range (Fig. 10). Furthermore, the deviation of the measurement of the porous sample viscoelastic properties depends on the measurement uncertainties of many parameters. This deviation is minimum around the resonance frequency of the transducer, i.e., $370 \mathrm{~Hz}$, and is relatively important above $500 \mathrm{~Hz}$. This is mainly due to the fact that the sought information $Z_{p}$ is part of the motional impedance [second term of Eq. (5)], which has a major influence on the resonance frequency of the transducer. Above and below this resonance, the electrical impedance $Z_{e}$ controls the global behavior of $Z_{v c}$ and $Z_{v c 0}$. Thus, a deviation on the estimation of the electrical parameters at low and high frequencies leads to an important deviation on the estimation of the viscoelastic parameter of the tested porous sample.

This is shown in Figs. 13 and 14 giving the contribution $C_{i}$ of parameters related to transducer and porous material to the global deviation of the viscoelastic parameters of the sample [Eq. (14)]. For both Young's modulus (Fig. 13) and loss factor (Fig. 14), the main influencing parameter is $r_{e}$ at low frequency and $L_{e}$ at high frequency. Around the resonance frequency of transducer $(370 \mathrm{~Hz}), L_{e}$ and $m$ are the most influent on Young's modulus and $\lambda$ for the loss factor, but the uncertainty is minimum is this region. It is also noticeable that the parameters relative to the porous acoustic behavior $\left(\phi, \sigma, \Lambda, \Lambda^{\prime}, \alpha_{\infty}, \rho_{1}\right)$ have a lesser contribution to the combined uncertainty.

According to the preceding remarks, it can be noticed that the valid frequency range of measurement, i.e., where the deviation is minimum, can be shifted in the high frequency range by shifting the resonance frequency of the
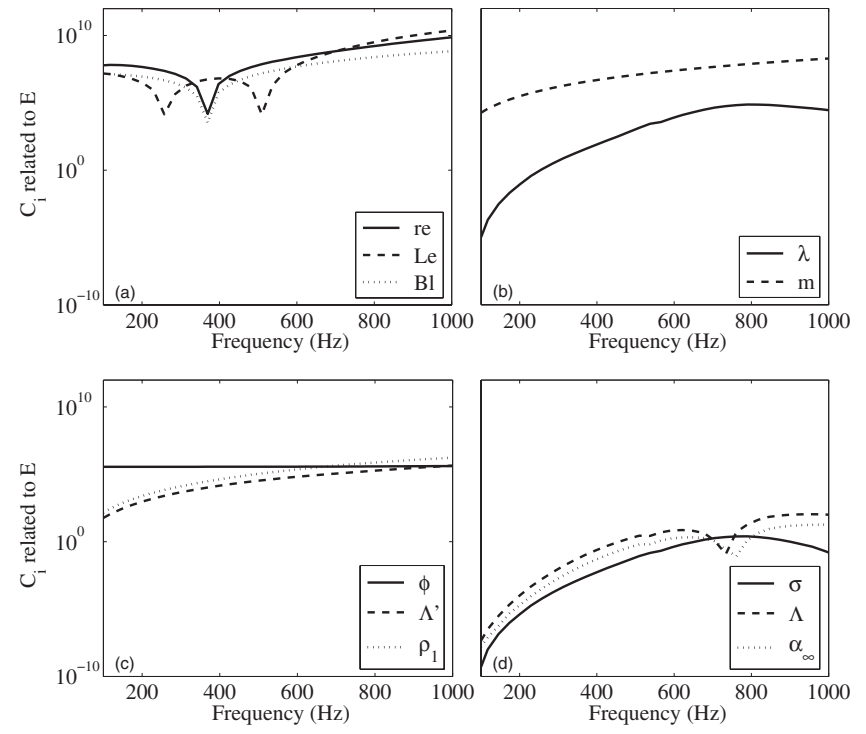

FIG. 13. Contribution of each parameter to the combined uncertainty of Young's modulus $E$.

transducer. This can be achieved by reducing the mass of the moving piston, decreasing the length of the bottom cavity, and decreasing the thickness of the porous sample.

\section{CONCLUSION}

A new device to determine the viscoelastic properties of porous materials has been developed. The apparatus, presented in Fig. 2, is based on an electrodynamic transducer used both as source and sensor. The setup is thus considerably simplified compared to a classical compression quasistatic method. Furthermore, this setup is based on a linear transducer that has been optimized to allow a static compression of $10 \mathrm{~mm}$, which cannot be performed with a classical shaker or loudspeakers. The transducer has also been designed so that the moving part behaves as a rigid solid body in an extended frequency range. Results given by this
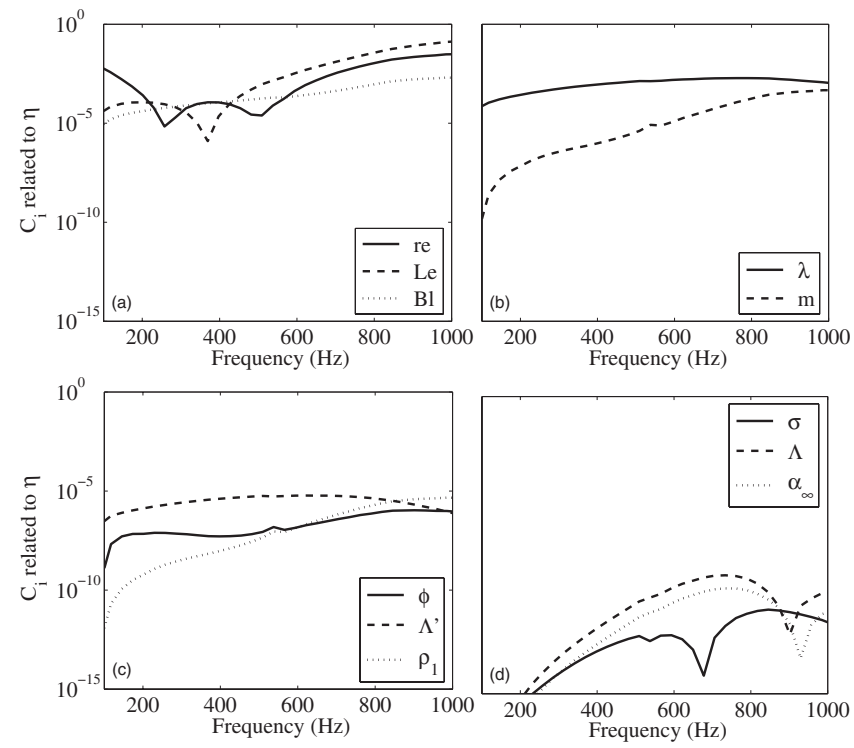

FIG. 14. Contribution of each parameter to the combined uncertainty of loss factor $\eta$. 
method are in good agreement with classical method and are valid for frequencies up to $500 \mathrm{~Hz}$. The air-pumping effect is also prevented by the use of a cavity, which avoids the overestimation of the loss factor. The method could be extended in the higher frequency range by optimizing the mass of the moving piston and the sizes of the cavities.

\section{ACKNOWLEDGMENTS}

The authors are grateful to the CNRS, Région Pays de la Loire, and the European Commission (CREDO Project, Contract No. 030814) for their financial support and to Benoît Merit for its contribution to design the magnetic part of the transducer.

${ }^{1}$ J. F. Allard and N. Atalla, Propagation of Sound in Porous Media: Modelling Sound Absorbing Materials, 2nd ed. (Wiley, Chichester, 2009).

${ }^{2}$ N. Dauchez, S. Sahraoui, and N. Atalla, J. Sound Vib. 265, 437 (2003).

${ }^{3}$ O. Doutres, N. Dauchez, and J. M. Génevaux, J. Acoust. Soc. Am. 121, 206 (2007)

${ }^{4}$ O. Doutres, N. Dauchez, J. M. Genevaux, and O. Dazel, J. Acoust. Soc. Am. 122, 2038 (2007).

${ }^{5}$ O. Doutres, N. Dauchez, J. M. Genevaux, and O. Dazel, Acta Acust. Acust. 95, 178 (2009).

${ }^{6}$ M. A. Biot, J. Appl. Phys. 33, 1482 (1962).

${ }^{7}$ L. Jaouen, A. Renault, and M. Deverge, Appl. Acoust. 69, 1129 (2008).

${ }^{8}$ E. Mariez and S. Sahraoui, Internoise Congress Proceedings, Liverpool, 1996, p. 951.

${ }^{9}$ N. Dauchez, M. Etchessahar, and S. Sahraoui, Proceedings of the Second Biot Conference on Poromechanics, 2002, p. 627.

${ }^{10}$ V. Tarnow, J. Acoust. Soc. Am. 118, 3672 (2005).

${ }^{11}$ M. Etchessahar, S. Sahraoui, L. Benyahia, and J. F. Tassin, J. Acoust. Soc. Am. 117, 1114 (2005).

${ }^{12}$ L. Boeckx, P. Leclaire, P. Khurana, C. Glorieux, W. Lauriks, and J. F. Allard, J. Acoust. Soc. Am. 117, 545 (2005).

${ }^{13}$ J. F. Allard, O. Dazel, J. Descheemaeker, N. Geebelen, L. Boeckx, and W.
Lauriks, J. Appl. Phys. 106, 014906 (2009).

${ }^{14} \mathrm{M}$. Etchessahar, "Caractérisation mécanique en basses fréquences des matériaux acoustiques," Ph.D. dissertation, Université du Maine, Le Mans, France, 2002.

${ }^{15}$ T. Pritz, J. Sound Vib. 72, 317 (1980).

${ }^{16}$ T. Pritz, J. Sound Vib. 106, 161 (1986).

${ }^{17}$ U. Ingard, Notes on Sound Absorption Technology (Noise Control Foundation, New York, 1994).

${ }^{18}$ H. J. Rice and P. Göransson, Int. J. Mech. Sci. 41, 561 (1999).

${ }^{19}$ O. Danilov, F. Sgard, and X. Olny, J. Sound Vib. 276, 729 (2004).

${ }^{20}$ O. Doutres, N. Dauchez, J.-M. Genevaux, and G. Lemarquand, J. Acoust. Soc. Am. 124, EL335 (2008).

${ }^{21}$ G. Lemarquand, IEEE Trans. Magn. 43, 3371 (2007).

${ }^{22}$ M. Berkouk, V. Lemarquand, and G. Lemarquand, IEEE Trans. Magn. 37, 1011 (2001).

${ }^{23}$ R. Ravaud, G. Lemarquand, and V. Lemarquand, J. Appl. Phys. 106, 034911 (2009)

${ }^{24}$ W. Klippel, J. Audio Eng. Soc. 54, 907 (2006).

${ }^{25}$ B. Merit, G. Lemarquand, and V. Lemarquand, IEEE Trans. Magn. 45, 2867 (2009)

${ }^{26}$ M. Remy, G. Lemarquand, B. Castagnede, and G. Guyader, IEEE Trans. Magn. 44, 4289 (2008).

${ }^{27}$ R. Ravaud and G. Lemarquand, Prog. Electromagn. Res. PIER 91, 53 (2009).

${ }^{28}$ R. Ravaud, M. Pinho, G. Lemarquand, N. Dauchez, J. M. Genevaux, B. Brouard, and V. Lemarquand, IEEE Trans. Magn. 45, 4388 (2009).

${ }^{29}$ M. Pinho, N. Dauchez, J. M. Genevaux, B. Brouard, and G. Lemarquand, Proceedings of the COBEM, International Congress of Mechanical Engineering, Gramados, RS, Brazil, 2009.

${ }^{30}$ A. N. Thiele, J. Audio Eng. Soc. 19, 382 (1971).

${ }^{31}$ R. H. Small, J. Audio Eng. Soc. 20, 798 (1972).

${ }^{32}$ R. H. Small, J. Audio Eng. Soc. 21, 11 (1973).

${ }^{33}$ Joint Committee for Guides in Metrology, Evaluation of Measurement Data-Guide to the Expression of Uncertainty in Measurement (JCGM 100:2008), Bur. Intl. Poids et Mesures (Sèvres, 2008).

${ }^{34}$ B. Castagnede, A. Aknine, M. Melon, and C. Depollier, Ultrasonics 36, 323 (1998).

${ }^{35}$ N. Dauchez, Ph.D. dissertation, Université du Maine, France, 1999. 